\title{
NOTE ON A THEOREM OF C. T. TAAM CONCERNING BOUNDED SOLUTIONS OF NONLINEAR DIFFERENTIAL EQUATIONS ${ }^{1}$
}

\author{
JOHN E. LAGNESE
}

1. In a recent paper [1], C. T. Taam considered the equation

$$
d X / d t+A X=F(t, X)
$$

where $t$ is real, $X$ and $F$ take values in a Banach space $B$ with norm $\|\cdot\|$, and $A$ is a closed linear operator from its domain $D(A) \subset B$ to $B$ satisfying $\mathrm{Cl} D(A)=B$ and

$$
\left\|(m I+A)^{-n}\right\| \leqq \beta(m+\alpha)^{-n}, \quad n=0,1, \cdots,
$$

for some pair $\alpha, \beta$ of positive numbers and all sufficiently large $m$. The function $F$ was required to satisfy the following conditions: ${ }^{2}$ $F$ maps $R \times B$ to $D(A)$ such that

(i) $F(t, X)$ is B.U.L. in $t$ and continuous in $X$ for almost all $t$.

(ii) $A F(t, X(t))$ is B.U.L. for every uniformly bounded continuous function $X(t)$ from $R$ to $B$.

(iii) There exists a function $\theta(t, \rho)$ from $R \times R^{+}$to $R^{+}$which is B.U.L. in $t$ and such that for fixed $\rho$ and each pair $X, Y$ with $\|X\| \leqq \rho$ and $\|Y\| \leqq \rho$ the Lipschitz condition

$$
\|F(t, X)-F(t, Y)\| \leqq \theta(t, \rho)\|X-Y\|
$$

holds for almost all $t$. Then if $\rho$ is such that

$$
\sup _{t \in R} \beta \int_{-\infty}^{0} e^{\alpha s \theta} \theta(t+s, \rho) d s<r<1,
$$

Taam showed that (1) has a unique solution $X(t)$ satisfying $\sup _{t \in R}\|X(t)\| \leqq \rho$. (See Theorems 1 and 7 of [1].) Taam's proof involves transforming (1) into an equivalent integral equation $X=T X$ and then using the contraction mapping principle to prove that the integral equation has a unique solution.

Received by the editors February 28, 1967 and, in revised form, January 28, 1968.

1 This research was supported by the National Science Foundation through Grant GP-4493.

${ }^{2}$ A solution of (1) is to be understood in the sense of [1, p. 872]. $R$ and $R^{+}$denote the real and nonnegative numbers respectively, and B.U.L. the Banach space of functions from $R$ to $B$ which are Bochner integrable on each unit interval, endowed with the uniform $L_{1}$-norm. See $[1$, p. 851]. 
In this note we prove a modified version of Taam's theorem. The essential difference is that under the assumptions we shall make on $F(t, X)$ the integral operator $T$ associated with (1) is no longer a contraction mapping. This difficulty is overcome by establishing precise estimates on the powers $T^{n}(n=1,2, \cdots)$ of $T$ (see Lemma, §2) and using these estimates to show that $T^{n}$ is, for sufficiently large $n$, a contraction mapping on the appropriate Banach space. Thus for some suitable integer $N, T^{N}$ will have a unique fixed point in this space and from this fact follows that $T$ itself has a unique fixed point as desired.

2. For our purposes it is convenient to write (1) in the form

$$
d X / d t+A X=F_{0}(t, X)+F_{1}(t, X) .
$$

Each of the functions $F_{i}(i=0,1)$ is required to satisfy conditions (i) and (ii) of the preceding section and also the Lipschitz condition (iii) with corresponding functions $\theta_{i}$ which are assumed to be independent of $\rho$. Thus we write $\theta_{i}(t)$ in place of $\theta_{i}(t, \rho)$ and assume

$$
\left\|F_{i}(t, X)-F_{i}(t, Y)\right\| \leqq \theta_{i}(t)\|X-Y\| \quad(i=0,1)
$$

for all $X, Y$ in $B$ and almost all $t$. The function $\theta_{0}$ is supposed to satisfy (3), i.e.

$$
\sup _{t \in R} \beta \int_{-\infty}^{t} \exp [\alpha(s-t)] \theta_{0}(s) d s<r<1 .
$$

Regarding $\theta_{1}$ we assume that it has support in some finite interval $[a, b]$ and is essentially bounded there, so that

$$
\text { ess } \sup _{t \in R} \theta_{1}(t) \leqq K<\infty, \quad \theta_{1}(t)=0 \quad \text { on } R-[a, b]
$$

Both the finite interval $[a, b]$ and the magnitude of $K$ are completely arbitrary. Clearly we may assume without loss of generality that $\theta_{1}$ is nondecreasing on $(-\infty, b]$. Under these conditions we can prove the following analog of Taam's theorem:

Theorem. Suppose the functions $F_{0}$ and $F_{1}$ satisfy (5), (6) and (7). Then (4) has one and only one solution $X(t)$ satisfying $\sup _{t \in R}\|X(t)\|$ $<\infty$.

We remark that the conclusions of Taam concerning the stability of the unique bounded solution of (1) extend also to our case with minor modifications, and we shall omit the details. Moreover, if $F_{0}$ and $F_{1}$ depend on a parameter $\lambda$ which is allowed to lie in some 
topological space, then under suitable conditions the bounded solutions $X(t, \lambda)$ of (4) can be shown to depend continuously on $\lambda$ (cf. $[1$, Theorem 2]). Finally, it is not difficult to verify that the unique bounded solution $X_{0}(t)$ of $d X / d t+A X=F_{0}(t, X)$ converges exponentially to the unique bounded solution $X(t)$ of $(4)$ as $t \rightarrow \infty$.

Proof of Theorem. Since the operator $A$ satisfies (2), it follows from the Hille-Phillips-Yosida Theorem that $-A$ is the infinitesimal generator of a semigroup $e(t,-A)$ of bounded linear operators from $B$ to $B$ defined and strongly continuous on $[0, \infty)$ and satisfying $\|e(t,-A)\| \leqq \beta e^{-\alpha t}$. Setting $e^{t A}=e(-t,-A)$ for $t \leqq 0, e^{t A}$ is then a semigroup defined and strongly continuous on $(-\infty, 0]$ such that $\left\|e^{t A}\right\| \leqq \beta e^{\alpha t}$.

Denote by $C(-\infty, \infty)$ the Banach space of uniformly bounded continuous functions $X(t)$ from $R$ to $B$ with norm $\|X\|_{\infty}=\sup _{t \in R}\|X(t)\|$. For $X \in C(-\infty, \infty)$ define

$$
(T X)(t)=\int_{-\infty}^{t} \exp [(s-t) A]\left[F_{0}(s, X(s))+F_{1}(s, X(s))\right] d s .
$$

According to [1, Lemma 5], $T$ is a mapping from $C(-\infty, \infty)$ into itself and each fixed point of $T$ in this space is a solution of (4). Conversely, any $C(-\infty, \infty)$ solution of (4) is a fixed point of $T$. To prove the theorem we have therefore to show that $T$ has a unique fixed point. For this we need the following lemma, whose proof is given in the next section.

Lemma. Suppose the functions $F_{0}$ and $F_{1}$ satisfy (5), (6) and (7). Let $T^{n}$ denote the nth iterate in $C(-\infty, \infty)$ of the operator defined by (8). Then for each positive integer $n$ and each pair $X(t), Y(t)$ in $C(-\infty, \infty)$ the following inequality holds:

$$
\begin{aligned}
\| T^{n} X & -T^{n} Y \|_{\infty} \\
& \leqq\|X-Y\|_{\infty} \sum_{k=0}^{n}\left(\begin{array}{l}
n \\
k
\end{array}\right) r^{n-k}\left(\frac{\beta K}{\alpha}\right)^{k}\left[e^{\alpha(b-a)}-\sum_{l=0}^{k-1} \frac{\alpha^{l}(b-a)^{l}}{l !}\right] .
\end{aligned}
$$

When $k=0$, the term in brackets is to be set equal to 1.

The operator $T$ has a unique fixed point in $C(-\infty, \infty)$ if for some positive integer $N, T^{N}$ has a unique fixed point there. Let $\gamma$ be a number satisfying $0<\gamma<1$. Since $r<1$ and $K>0$ we can choose $m>0$ so large that $r+\beta / m<1$ and $K m \geqq \alpha$. Having picked $\gamma$ and $m$, we can find a positive integer $N$ such that $(r+\beta / m)^{n} \leqq \gamma e^{-K m(b-a)}$ whenever $n \geqq N$. Then for $n \geqq N$ and any pair $X(t), Y(t)$ in $C(-\infty, \infty)$ we have from (9) 


$$
\begin{aligned}
\| T^{n} X & -T^{n} Y \|_{\infty} \\
& \leqq\|X-Y\|_{\infty} \sum_{k=0}^{n}\left(\begin{array}{l}
n \\
k
\end{array}\right) r^{n-k}\left(\frac{\beta}{m}\right)^{k}\left(\frac{K m}{\alpha}\right)^{k}\left[e^{\alpha(b-a)}-\sum_{l=0}^{k-1} \frac{\alpha^{l}(b-a)^{l}}{l !}\right] \\
& \leqq\|X-Y\|_{\infty}\left(r+\frac{\beta}{m}\right)^{n} \exp [K m(b-a)] \leqq \gamma\|X-Y\|_{\infty} .
\end{aligned}
$$

Thus $T^{N}$ is a contraction mapping on $C(-\infty, \infty)$ and, therefore, has a unique fixed point $X(t)$. The Theorem is now established.

3. Proof of the lemma. For $X(t), Y(t)$ in $C(-\infty, \infty)$ and $n=1,2, \cdots$, we have from (5) and (8)

$$
\begin{aligned}
\|\left(T^{n} X\right)(t)- & \left(T^{n} Y\right)(t) \| \\
\leqq & \beta \int_{-\infty}^{t} e^{\alpha(s-t)}\left[\theta_{0}(s)+\theta_{1}(s)\right]\left\|\left(T^{n-1} X\right)(s)-\left(T^{n-1} Y\right)(s)\right\| d s \\
\leqq & \beta^{n}\|X-Y\|_{\infty} \int_{-\infty}^{t} \exp \left[\alpha\left(s_{1}-t\right)\right]\left[\theta_{0}\left(s_{1}\right)+\theta_{1}\left(s_{1}\right)\right] \\
& \cdot \int_{-\infty}^{s_{1}} \exp \left[\alpha\left(s_{2}-s_{1}\right)\right]\left[\theta_{0}\left(s_{2}\right)+\theta_{1}\left(s_{2}\right)\right] \ldots \\
& \cdots \int_{-\infty}^{s_{n-1}} \exp \left[\alpha\left(s_{n}-s_{n-1}\right)\right]\left[\theta_{0}\left(s_{n}\right)+\theta_{1}\left(s_{n}\right)\right] d s_{n} \cdots d s_{1} .
\end{aligned}
$$

The coefficient of $\|X-Y\|_{\infty}$ is expressible as the sum of $2^{n}$ iterated integrals, each of the form

$$
\begin{array}{r}
\beta^{n} \int_{-\infty}^{t} \exp \left[\alpha\left(s_{1}-t\right)\right] \theta_{k_{1}}\left(s_{1}\right) \int_{-\infty}^{s_{1}} \exp \left[\alpha\left(s_{2}-s_{1}\right)\right] \theta_{k_{2}}\left(s_{2}\right) \cdots \\
\cdots \int_{-\infty}^{s_{n-1}} \exp \left[\alpha\left(s_{n}-s_{n-1}\right)\right] \theta_{k_{n}}\left(s_{n}\right) d s_{n} \cdots d s_{1}
\end{array}
$$

with each $k_{i}=0$ or 1 . Among these $2^{n}$ integrals, there are $\left(\begin{array}{l}n \\ k\end{array}\right)$ in which $\theta_{0}$ appears exactly $n-k$ times. Each of these is not greater than

$$
\begin{gathered}
\beta^{k} r^{n-k} \int_{a}^{b} \exp \left[\alpha\left(s_{1}-a\right)\right] \theta_{1}\left(s_{1}\right) \int_{a}^{s_{1}} \exp \left[\alpha\left(s_{2}-s_{1}\right)\right] \theta_{1}\left(s_{2}\right) \cdots \\
\cdots \int_{a}^{s_{k-1}} \exp \left[\alpha\left(s_{k}-s_{k-1}\right)\right] \theta_{1}\left(s_{k}\right) d s_{k} \cdots d s_{1} .
\end{gathered}
$$

One obtains the bound (11) from (6), (7) and the following observa- 
tion: If $\theta(t) \geqq 0$ is a nondecreasing function on $(-\infty, b]$, then for each positive integer $k$, the function

$$
\begin{gathered}
f_{k}(s)=\int_{-\infty}^{e} \exp \left[\alpha\left(s_{1}-s\right)\right] \theta\left(s_{1}\right) \int_{-\infty}^{s_{1}} \exp \left[\alpha\left(s_{2}-s_{1}\right)\right] \theta\left(s_{2}\right) \cdots \\
\cdots \int_{-\infty}^{s_{k-1}} \exp \left[\alpha\left(s_{k}-s_{k-1}\right)\right] \theta\left(s_{k}\right) d s_{k} \cdots d s_{1}
\end{gathered}
$$

is also nondecreasing on $(-\infty, b]$. This follows by writing

$$
f_{k}(s)=\int_{-\infty}^{s} e^{\alpha(t-s)} \theta(t) f_{k-1}(t) d t
$$

and noting that

$$
f_{1}(s)=\int_{-\infty}^{s} e^{\alpha(t-s)} \theta(t) d t=\int_{-\infty}^{0} e^{\alpha t} \theta(t+s) d t,
$$

is $\geqq 0$ and nondecreasing on $(-\infty, b]$. Now since $\theta_{1}$ is nondecreasing on $(-\infty, b]$, one can estimate, for example, an iterated integral of the form

$$
I_{01}=\int_{-\infty}^{t} \exp \left[\alpha\left(s_{1}-t\right)\right] \theta_{0}\left(s_{1}\right) \int_{-\infty}^{s} \exp \left[\alpha\left(s_{2}-s_{1}\right)\right] \theta_{1}\left(s_{2}\right) d s_{2} d s_{1},
$$

as follows: For $t \leqq b$ we have

$$
\begin{aligned}
I_{01} & \leqq \int_{-\infty}^{t} \exp \left[\alpha\left(s_{1}-t\right)\right] \theta_{0}\left(s_{1}\right) \int_{-\infty}^{b} \exp \left[\alpha\left(s_{2}-b\right)\right] \theta_{1}\left(s_{2}\right) d s_{2} d s_{1} \\
& \leqq \int_{a}^{b} \exp \left[\alpha\left(s_{2}-b\right)\right] \theta_{1}\left(s_{2}\right) d s_{2} \int_{-\infty}^{t} \exp \left[\alpha\left(s_{1}-t\right)\right] \theta_{0}\left(s_{1}\right) d s_{1} \\
& \leqq \frac{r}{\beta} \int_{a}^{b} \exp \left[\alpha\left(s_{2}-a\right)\right] \theta_{1}\left(s_{2}\right) d s_{2}
\end{aligned}
$$

remembering that $\theta_{1}(s)=0$ for $s \in R-[a, b]$. For $t>b$ we have.

$$
\begin{aligned}
I_{01} \leqq & \int_{-\infty}^{b} \exp \left[\alpha\left(s_{1}-t\right)\right] \theta_{0}\left(s_{1}\right) \int_{-\infty}^{b} \exp \left[\alpha\left(s_{2}-b\right)\right] \theta_{1}\left(s_{2}\right) d s_{2} d s_{1} \\
& +\int_{b}^{t} \exp \left[\alpha\left(s_{1}-t\right)\right] \theta_{0}\left(s_{1}\right) \int_{-\infty}^{s_{1}} \exp \left[\alpha\left(s_{2}-s_{1}\right)\right] \theta_{1}\left(s_{2}\right) d s_{2} d s_{1} .
\end{aligned}
$$

Since $s_{1} \geqq b$ in the second integral 


$$
\begin{aligned}
I_{01} & \leqq \int_{a}^{b} \exp \left[\alpha\left(s_{2}-b\right)\right] \theta_{1}\left(s_{2}\right) d s_{2}\left(\int_{-\infty}^{b}+\int_{b}^{t}\right) \exp \left[\alpha\left(s_{1}-t\right)\right] \theta_{0}\left(s_{1}\right) d s_{1} \\
& \leqq \frac{r}{\beta} \int_{a}^{b} \exp \left[\alpha\left(s_{2}-a\right)\right] \theta_{1}\left(s_{2}\right) d s_{2}
\end{aligned}
$$

again in agreement with (11). Similar considerations can be applied to the general case to prove that (11) is indeed an upper bound for (10).

Finally, (11) is bounded above by

$$
\begin{aligned}
(\beta K)^{k} r^{n-k} \int_{a}^{b} \exp \left[\alpha\left(s_{1}-a\right)\right] & \int_{a}^{s_{1}} \exp \left[\alpha\left(s_{2}-s_{1}\right)\right] \cdots \\
& \cdots \int_{a}^{s_{k-1}} \exp \left[\alpha\left(s_{k}-s_{k-1}\right) d s_{k} \cdots d s_{1}\right] \\
& =\left(\frac{\beta K}{\alpha}\right)^{k} r^{n-k}\left[e^{\alpha(b-a)}-\sum_{l=0}^{k-1} \frac{\alpha^{l}(b-a)^{l}}{l !}\right],
\end{aligned}
$$

and therefore

$$
\begin{aligned}
& \left\|\left(T^{n} X\right)(t)-\left(T^{n} Y\right)(t)\right\| \\
& \leqq\|X-Y\|_{\infty} \sum_{k=0}^{n}\left(\begin{array}{l}
n \\
k
\end{array}\right) r^{n-k}\left(\frac{\beta K}{\alpha}\right)^{k}\left[e^{\alpha(b-a)}-\sum_{l=0}^{k-1} \frac{\alpha^{l}(b-a)^{l}}{l !}\right],
\end{aligned}
$$

for all $t \in R$, as was to be proved.

\section{REFERENCE}

1. C. T. Taam, Stability, periodicity and almost periodicity of solutions of nonlinear differential equations in Banach spaces, J. Math. Mech. 15 (1966), 849-876.

GEORGETOWN UNIVERSITY 